\title{
Forecasting ozone concentrations in the east of Croatia using nonparametric Neural Network Models
}

\author{
Elvira Kovač-Andrićc ${ }^{1, *}$, Alaa Sheta ${ }^{2}$, Hossam Faris ${ }^{3}$ and Martina Šrajer Gajdošik ${ }^{1}$ \\ ${ }^{1}$ Department of Chemistry, University of J. J. Strossmayer, Cara Hadrijana 8/A, Osijek 31000, Croatia. \\ ${ }^{2}$ Computers and Systems Department, Electronics Research Institute, Giza, Egypt. \\ ${ }^{3}$ King Abdullah II School for Information Technology, The University of Jordan, Amman, Jordan. \\ ${ }^{*}$ Corresponding author.e-mail: eakovac@kemija.unios.hr
}

\begin{abstract}
Ozone is one of the most significant secondary pollutants with numerous negative effects on human health and environment including plants and vegetation. Therefore, more effort is made recently by governments and associations to predict ozone concentrations which could help in establishing better plans and regulation for environment protection. In this study, we use two Artificial Neural Network based approaches (MPL and RBF) to develop, for the first time, accurate ozone prediction models, one for urban and another one for rural area in the eastern part of Croatia. The evaluation of actual against the predicted ozone concentrations revealed that MLP and RBF models are very competitive for the training and testing data in the case of Kopački Rit area whereas in the case of Osijek city, MLP shows better evaluation results with $9 \%$ improvement in the correlation coefficient. Furthermore, subsequent feature selection process has improved the prediction power of RBF network.
\end{abstract}

\section{Introduction}

Atmospheric pollutants in the boundary layer of the atmosphere have a significant impact on the overall life on Earth and that includes the impact on human health, life of plants and durability of some materials (Alexis et al. 2004; Percy and Ferretti 2004; Yang and Omaye 2009). Technology development and growth of urban areas lead to the need for more effective modes of transportation. Due to increased traffic mobility in the urban environment, pollution as such grows as well. The composition of air pollution often varies depending on the distance of roads, flow of traffic, industry, agricultural activities, burning forests and other sources of pollution. Particulate matter (PM) are the primary pollutants that come into the atmosphere by direct emission from different sources. In their composition, PM may contain metals such as aluminium, chromium, nickel and lead; their potential health risk should not be ignored. Atmospheric PM that are commonly observed in recent studies are $\mathrm{PM}_{10}$ (aerodynamic diameter $<10 \mu \mathrm{m}$ ) which can easily penetrate into the deeper parts of the respiratory system (Katsouyanni et al. 2001; Pope et al. 2002; Prajapati and Triphati 2008).

One of the most significant pollutants is ozone. Ozone is a secondary pollutant formed by series of reactions of primary pollutants (volatile organic compounds and nitrogen oxides) and an important oxidant at ground level in the atmospheric boundary layer (Sahu 2012; Sahu and Saxena 2015).

During favourable meteorological conditions, photochemical production of ozone occurs (Kalabokas et al. 2007; Sánchez-Lorenzo et al. 2008; Paoletti 2009). Different pollutants, especially ozone and

Keywords. Ozone; $\mathrm{PM}_{10}$; rural and urban area; prediction models; artificial neural networks. 
PM, lead to adverse health effects such as bronchitis, asthma, and other cardio-pulmonary problems and even premature death (Stedman 2004; AlebićJuretić et al. 2007; Chen et al. 2010). Apart from the negative impact on respiratory system, atmospheric pollutants also affect birth outcomes. For example, fetal exposure to $\mathrm{PM}_{10}$ and ozone is associated with reduction in birth weight (Salam et al. 2005). Furthermore, among many different effects on environment, ozone also affects vegetation resulting in growth changes, yield losses and reduced seed production and quality (Bytnerowicz et al. 2007; Cape 2008; Wittig et al. 2009).

Due to aforementioned negative health and environmental impacts of the ozone, efforts were made to develop accurate prediction models for ozone concentration. Accurate and robust models can effectively help in designing environment and health strategies and plans. In previous works, ozone prediction models include wide range of statistical and machine learning models. Among them, Artificial Neural Network (ANN) models showed higher accuracy and capability in capturing the dynamics of the complex problem in comparison with other statistical based approaches like Autoregressive Moving Average (ARMA) and linear regression models (Prybutok et al. 2000; Pastor-Bárcenas et al. 2005; Agirre et al. 2012; Faris et al. 2014).

Until now, there was no study on predicting the concentration of surface ozone in the rural and urban areas of eastern Croatia. The aim of our study is to develop an accurate ozone prediction model using two artificial neural network models, the Multilayer Perceptron (MLP) network and the Radial Basis Function (RBF) network. The two developed models are evaluated and compared based on real data measured for Osijek city and Kopački Rit. The surface ozone and meteorological variables are measured in the city of Osijek and Kopački Rit for a period of 8 months in 2012. The measured data are discussed in relation to the EU guidelines on pollution in ambient air.

\section{Methods}

\subsection{Artificial neural networks}

ANN are parallel distributed processing systems composed of simple processing units called neurons. These simple processing units are capable of developing a complex mathematical function that map a relationship between input and output space. Neurons are always organized in one or more layers. The neurons in each layer are interconnected with the neurons in the next layer by a massive number of connections. These connections are associated with weights. These weights are varying based on the acquired knowledge for each developed model nature. The models developed based on Feedforward Neural Networks (FFNN) or RBF network are always nonparametric models since the ANN's weight has no definite meaning relating to the problem in hand. It is always the case that ANN is used to estimate the input-output mapping function with the best possible accuracy that minimizes the error difference between the inputoutput pairs. The MLP and RBF were used in many types of applications such as nonlinear mapping, function approximation and pattern recognition, although they have different structure and method of learning. In this paper we aim to create a comparison between MLP network and a RBF network in modelling the dynamics of ozone concentration based on number of metrological attributes. In the following two subsections, we represent the MLP and RBF networks applied in this work.

\subsubsection{Multilayer perceptron (MLP) neural network}

The multilayer perceptron neural network (MLP) is a common mathematical model and a type of FFNN. The basic component of MLP is 'neuron' which is a simple processing element. Neurons are arranged in layers and each layer is fully connected with the next one by means of weights. An example of MLP is depicted in figure 1.

MLP networks process information as follows: given an input value $x_{i}$ at neuron $i$ and a set of corresponding weights between the input and hidden neuron $w_{i j}$, the output of each neuron in the hidden layer is calculated in two steps, first by the following summation function:

$$
S_{i}=\sum_{i=0}^{n-1} w_{i j} x_{i}-\beta_{1}
$$

where $\beta_{1}$ is a threshold and $n$ is the number of neurons in the hidden layer.

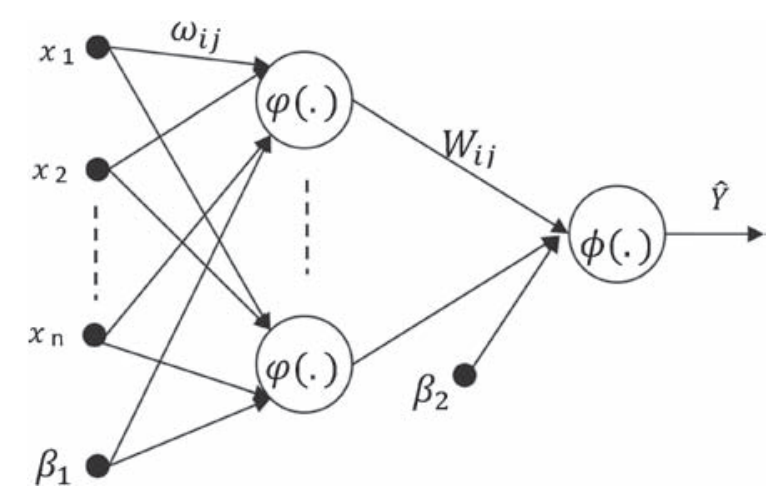

Figure 1. MLP structure. 
Then the output of the summation function $S_{i}$ goes as input for an activation function

$$
\varphi_{i}\left(S_{i}\right)=\frac{1}{1+e^{-S_{i}}} .
$$

The output of each neuron in the output layer is computed as given in equation (4). $f_{l}$ is the activation function of neuron $l$ in the output layer which is usually a linear function and $\beta_{2}$ is a threshold.

$$
\phi_{l}(x)=f_{l}\left(\sum_{j=0}^{m} W_{j l} \varphi_{j}(x)-\beta_{2}\right) .
$$

MLP is trained by updating all connection weights until a predetermined level of error is reached. In our work we use the backpropagation algorithm for training the MLP network. Backpropagation algorithm is one of the most common techniques to train the feedforward networks. The algorithm uses the steepest gradient descent method to update the weights in order to minimize the error.

\subsubsection{Radial basis function (RBF) network}

$\mathrm{RBF}$ is another common type of feedfoward neural networks. This network consists of three layers including the input layer. The main difference between RBF and MLP networks is the way that the hidden layer performs its computation. In RBF, each neuron in the hidden layer computes the Euclidean distance between an input vector $u$ and a point $c_{i}$ stored in the neuron commonly referred to as a 'centre vector' or 'prototype'. The output $\phi_{i}$ of each hidden neuron $i$ is then computed by a basis function. Typically, Gaussian function is used as follows:

$$
\phi_{i}(u)=e^{-\left\|u-c_{i}\right\|^{2} / 2 \sigma_{i}^{2}}
$$

where $\|\ldots\|$ is the Euclidean norm, $u$ is the centre of the neuron $i, \sigma_{i}$ is the width (radius) and $\phi(x)$ is the output of the neuron. The output of each neuron in the output layer is a linear combination of the outputs from the hidden layer and it is computed as follows:

$$
f_{j}(u)=\sum_{i=1}^{m} w_{i j} \phi_{i}(u)
$$

where $m$ is the number of neurons in the hidden layer and $w_{i j}$ is the connection weight between the hidden neuron $i$ and the output neuron $j$.

In RBF, the objective of the training algorithm is to minimize the error which is the difference between the actual values and the estimated ones by finding the best set of centres and widths of the RBFs and the weights connecting the hidden neurons and the output layer. Traditionally, training
$\mathrm{RBF}$ is carried out in two stages independently. In the first stage, centres and weights are found using some technique like a clustering algorithm. While in the second stage, the weights are optimized. Some of the advantages of RBF network are the fast training algorithms while maintaining good interpolation capability.

\subsection{Model reduction based feature selection}

In data mining, feature selection is a process of choosing $N$ features out of $M$ available features in the given dataset where $N<M$ so that the value of some determine evaluation criteria is optimized over the set of all possible feature subsets available. The goal of the feature selection process is to eliminate redundant, noisy and unrepresentative features and consequently reduce the training time of the model and its complexity. Generally, there are two main types of feature selection methods: filter based and wrapper based. Filter-based approaches depend on the statistical properties of the features. These types are fast and do not require tremendous processing time. However, they do not incorporate any training model and therefore ignore the effect of selected features on the final classification or regression model.

In contrast to filter-based approaches, wrapper approaches incorporate an induction model while searching for the best set of features (Kohavi and John 1997). Therefore, in this work, we adopt the wrapper feature selection method in order to select the best subset of features to achieve the highest prediction accuracy with our developed model.

As shown in figure 2, this method consists of three main components: the search algorithm, the evaluation function and the induction algorithm. The search algorithm searches the space of all possible feature subsets and evaluates each selected subset based on the predictive power of the induction algorithm after training it using these subsets. The advantage of this method is that it takes into account feature dependencies and it incorporates the interaction between the features and the model (Saeys et al. 2007). On the other hand, the main disadvantage of this method is that it is computationally expensive. However, this is not a problem in our case since the number of input features in the investigated dataset is small.

\subsection{Performance evaluation}

In order to check the performance of the developed models, correlation coefficient $\left(R^{2}\right)$, mean squares error (MSE), mean absolute error (MAE), root mean square error (RMSE) and varianceaccounted-for (VAF) are measured. These performance criteria are used to measure how close the 


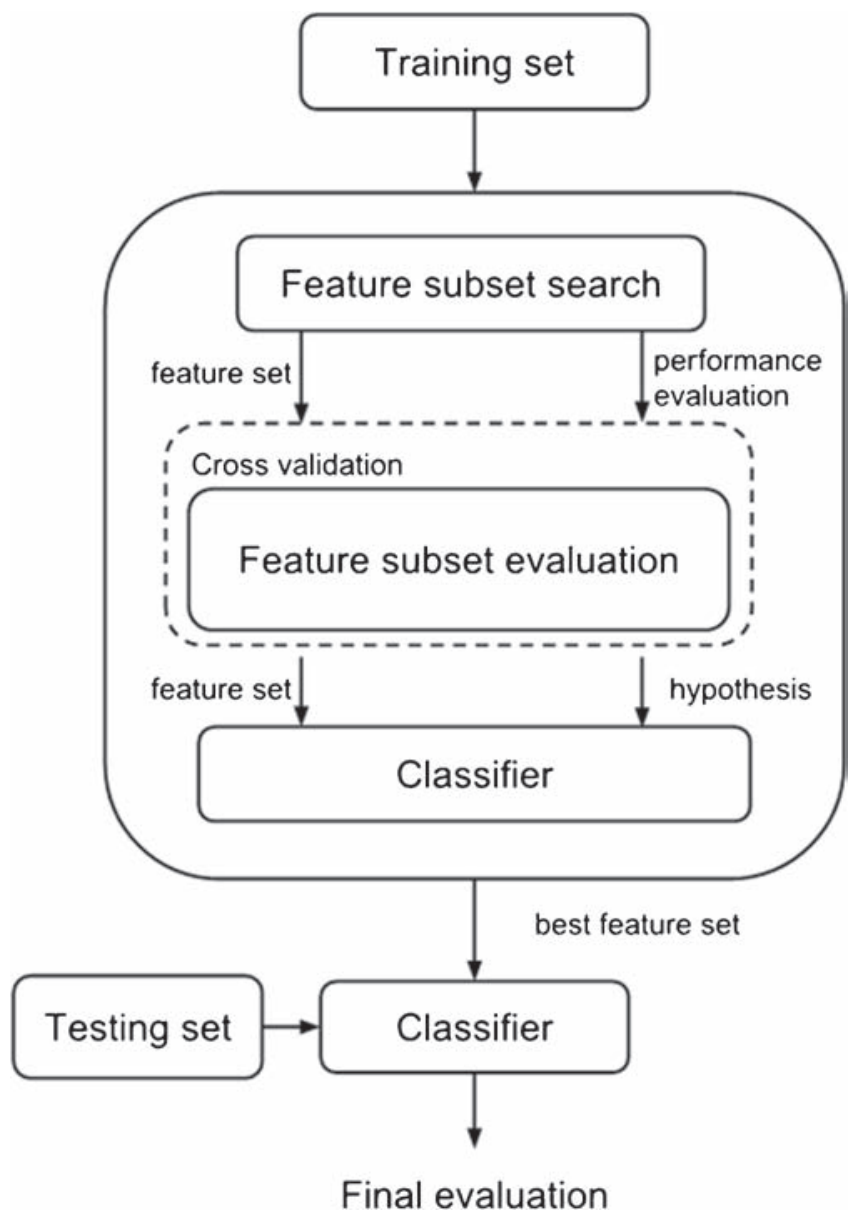

Figure 2. Framework of wrapper feature selection.

computed ozone concentrations to the real measured values. $R^{2}$, MSE and MAE and RMSE are computed as:

$$
\begin{gathered}
R^{2}=\frac{\sum_{i=1}^{n}\left(y_{i}-\bar{Y}_{i}\right)^{2}-\sum_{i=1}^{n}\left(y_{i}-\hat{y}_{i}\right)^{2}}{\sum_{i=1}^{n}\left(y_{i}-\bar{y}_{i}\right)^{2}} \\
\text { MSE }=\frac{1}{n} \sum_{i=1}^{n}\left(y_{i}-\hat{y}_{i}\right)^{2} \\
\text { MAE }=\frac{1}{n} \sum_{i=1}^{n}\left|y_{i}-\hat{y}_{i}\right| \\
\text { RMSE }=\sqrt{\frac{1}{n} \sum_{i=1}^{n}\left(y_{i}-\hat{y}_{i}\right)^{2}} \\
\text { VAF }=\left[1-\frac{\operatorname{var}(y-\hat{y})}{\operatorname{var}(y)}\right] \cdot 100 \%
\end{gathered}
$$

where $y$ is the real actual value, $\hat{y}$ is the estimated target value. $n$ is the total number of measurements.

\subsection{Site and data description}

The eastern part of Croatia is prominently an agricultural area. The largest urban centre there is the city of Osijek. A few kilometres northeast of Osijek lies Nature Reserve Kopački Rit creating the possibility of atmospheric interactions. The Nature Reserve Kopački Rit is one of the largest natural marshlands in Europe with area of almost $240 \mathrm{~km}^{2}$. Preservation of Kopački Rit is of great importance, because it is among the few natural marshlands in Europe that remained intact. For this reason, it was recognized in UNESCO and nominated for the World Heritage List (UNESCO 2001; Ministry of Environmental Protection 2002).

Ozone volume mixing ratios have been measured and analysed in the city of Osijek. Monitoring site is located at the northern boundary of the city, close to the river Drava and approximately $15 \mathrm{~m}$ above the ground. Osijek is the largest urban centre in eastern Croatia located at $45.32^{\circ} \mathrm{N}, 18.44^{\circ} \mathrm{E}$ (with a population of approx. 130,000). Since it has no significant industry, precursor emissions are mainly due to traffic. The measurements took place during the period of April-November of 2012, using a commercial instrument Ansyco O341M based on UV absorption photometry. The instrument was regularly checked and calibrated. The data have been recorded every $3 \mathrm{~min}$ and stored in data logger for further computer processing. Air pollution quality data were obtained from the Ministry of Environmental and Nature Protection of Croatia which is monitoring the atmospheric pollutants (nitrogen dioxide $\left(\mathrm{NO}_{2}\right)$, sulphur dioxide $\left(\mathrm{SO}_{2}\right)$, benzene $\left(\mathrm{C}_{6} \mathrm{H}_{6}\right)$, carbon monoxide $(\mathrm{CO})$ and $\left.\mathrm{PM}_{10}\right)$ using monitoring station located about $100 \mathrm{~m}$ to the east of the ozone measurement site. Meteorological data were obtained from the Meteorological and Hydrological Service of Croatia. They have monitoring site for meteorological parameters at Klisa (airport cca. $20 \mathrm{~km}$ to the southeast of our monitoring site).

The district of Kopački Rit Nature Park is located mostly in the low areas between the Drava and Danube rivers. Geographically, Kopački Rit extends between $45.53^{\circ}$ and $45.78^{\circ} \mathrm{N}$ and $18.75^{\circ}$ and $18.98^{\circ} \mathrm{E}$. The altitude in Baranja does not exceed $250 \mathrm{~m}$, and the whole area of the Nature Park lies between $78 \mathrm{~m}$ (bottom lakes) and $86 \mathrm{~m}$ in altitude. The monitoring site was located at the village of Tikveš (with a population of approximately 30) that lies in the centre of the northern part of the Nature Park $\left(45.67^{\circ} \mathrm{N}, 18.85^{\circ} \mathrm{E}\right)$, at an altitude of $82 \mathrm{~m}$ above sea level. The measuring site is a part of national network for continuous air quality monitoring set up by the Croatian Ministry of Environmental Protection. 
The atmospheric pollutants $\left(\mathrm{O}_{3}\right.$ and $\left.\mathrm{PM}_{10}\right)$ are measured with automatic analyzer (UV absorption for ozone and absorption of $\beta$ radiation for $\mathrm{PM}_{10}$ ). All instruments have been regularly checked and calibrated. The data have been recorded every $10 \mathrm{~min}$ and converted to hourly values. Data analysis is commonly based on hourly average.

\section{Results and discussion}

\subsection{Comparison of ozone and $P M_{10}$ in urban and rural areas}

In urban area, ambient air is a dynamic mixture of pollutants coming from numerous sources including traffic, industry, and combustion as well as neighbouring rural sources such as agriculture activities and burning forests. Between these pollutants, ozone and $\mathrm{PM}_{10}$, that affect human health and vegetation, stand out.

The standard value in use in European countries for the tropospheric ozone air quality is $120 \mu \mathrm{g} / \mathrm{m}^{3}$. That value may not exceed on more than 25 days per calendar year averaged over 3 years (Directive 2002/3/E; Directive 2008/50/EC). During the measured period, the above-mentioned standard value of ozone was not exceeded neither in rural nor in urban areas. Interestingly, in the beginning of measurement (during the month of April) daily concentration values for ozone differ significantly among monitoring stations (figure 3). Higher concentrations of ozone are recorded in rural area. Such results may be due to the increased production of ozone caused by emissions of biogenic volatile organic compounds. We presume that this pattern is likely to be attributed almost exclusively to physical processes in atmosphere like speed and wind direction or dry and wet deposition as the influence of chemistry in this nonindustrial and tourist resort area is unlikely (Kovač-Andrić et al. 2013). Low ozone concentrations in urban area during April can be a result of titration of ozone by nitrogen oxides. During May and all through the rest of the measured period, average daily concentrations of ozone show similar patterns in both urban and rural areas, slightly higher in urban area.

The daily averages from $\mathrm{PM}_{10}$ concentrations are shown in figure 4 . The variation of daily $\mathrm{PM}_{10}$ concentration was in the ranges of $4.9-96.8 \mu \mathrm{g} / \mathrm{m}^{3}$ in Osijek and $3.4-44.4 \mu \mathrm{g} / \mathrm{m}^{3}$ in Tikveš. The variation trend of $\mathrm{PM}_{10}$ at these locations in the first 50 days was similar, with lowest values in Tikveš.

According to EU guidelines (Directive 2008/ $50 / \mathrm{EC}$ ) daily limit for $\mathrm{PM}_{10}$ is $50 \mu \mathrm{g} / \mathrm{m}^{3}$ (daily value) and should not be exceeded on more than 35 days per calendar year. By calculating daily average $\mathrm{PM}_{10}$ concentrations in Osijek, it was found that the value of $50 \mu \mathrm{g} / \mathrm{m}^{3}$ was exceeded in 26 days during the measured period. Further, we calculated estimated daily average $\mathrm{PM}_{10}$ concentrations for the calendar year, and this value is 39.4 days. Accordingly, this estimated value exceeds the critical level of 35 days per calendar year. The values in Tikveš exceeded the daily limit only in 2 days of measured period. Whereas the estimated daily average value is 3.03 days.

\subsection{Daily ozone forecasting models}

In our experiments we developed two daily ozone forecasting models for Osijek city and the area of Kopački Rit in the east of Croatia. We used the MLP and RBF neural networks for developing the forecasting models. The inputs of the models are daily measurements of the meteorological

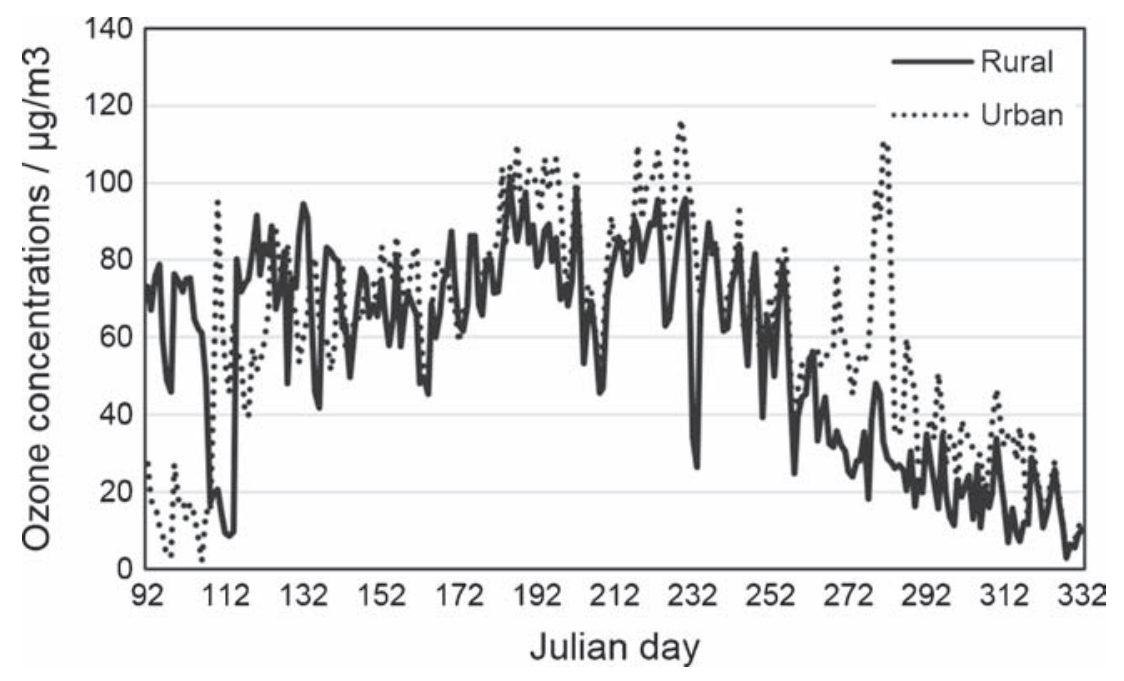

Figure 3. Daily average ozone concentrations in the rural (Tikveš) and urban (Osijek) areas. 


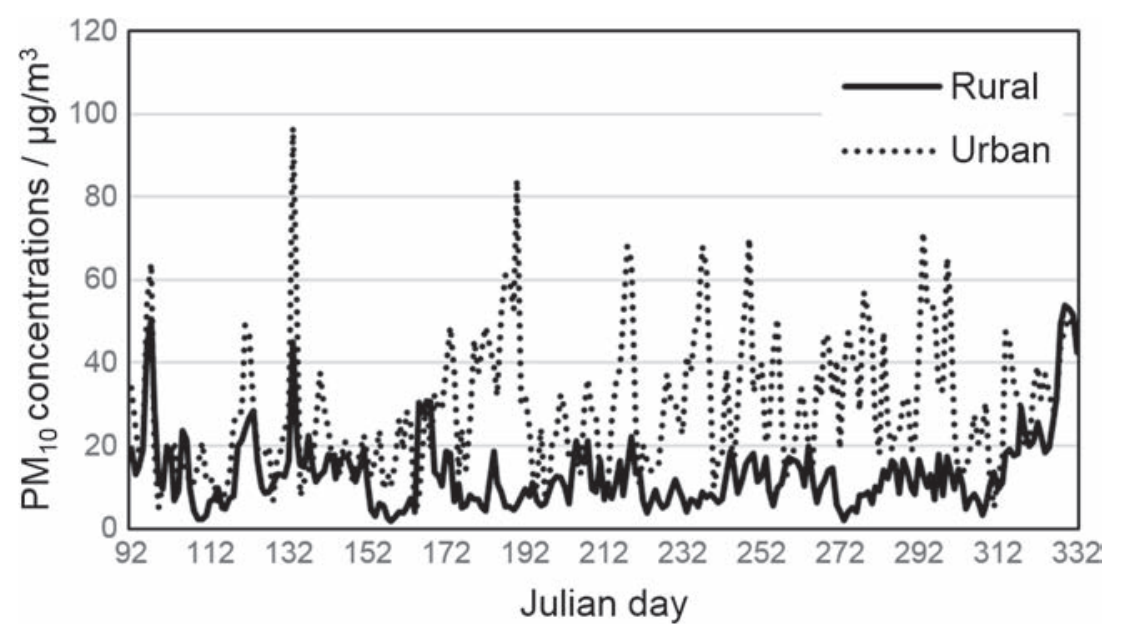

Figure 4. Daily average $\mathrm{PM}_{10}$ concentrations in the rural (Tikveš) and urban (Osijek) areas.

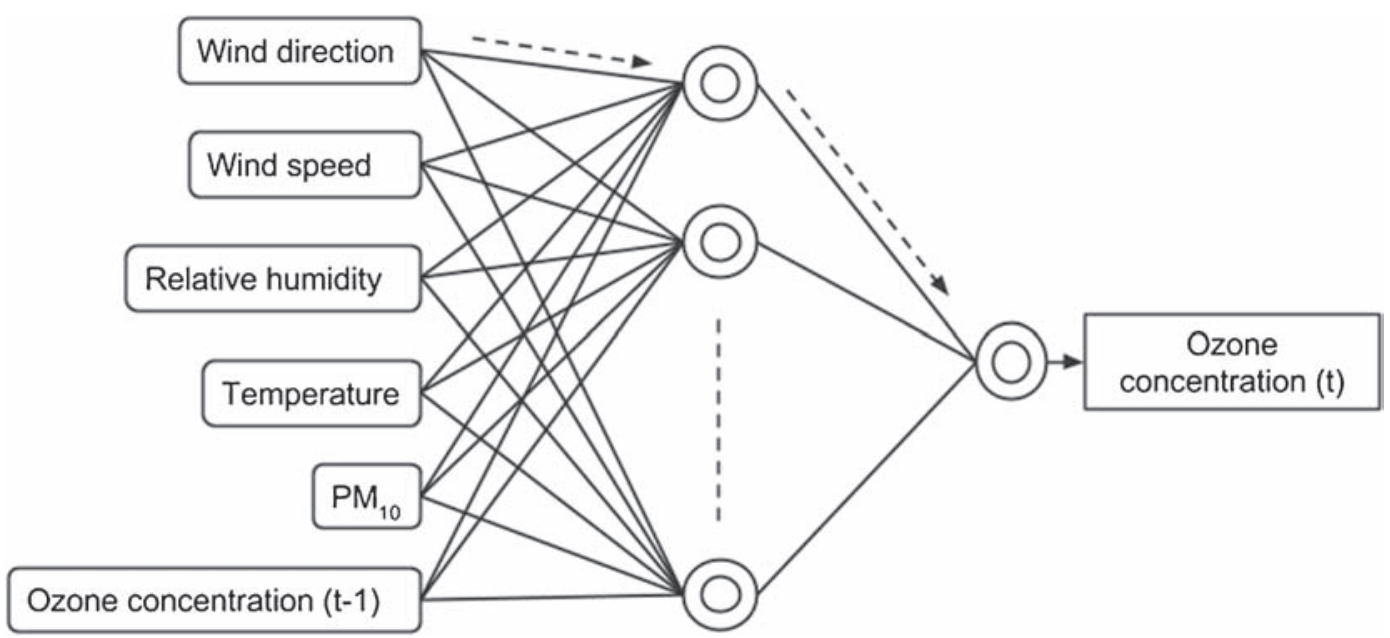

Figure 5. Proposed input/output ANN based model.

conditions; temperature, relative humidity, wind speed and wind direction and the pollutants $\mathrm{PM}_{10}$ and ozone concentration of the previous day. The proposed input/output ANN based model is shown in figure 5.

Before developing our forecasting models, we prepared the data by calculating the average of the measured meteorological conditions, $\mathrm{PM}_{10}$ and $\mathrm{O}_{3}$ for each day starting from April 2012 until November 2012 for the two studied areas. The dataset for first 25 days of each month were collected together and formed the training set while the last 5 days of each month formed the testing set. The size of each training dataset is 199 data points and the size of the testing dataset is 41 points.

Based on the prepared training dataset, we trained the MLP and RBF networks. Empirically, it was found that the best MLP performance could be obtained by tuning the parameters as shown
Table 1. MLP tuning settings.

\begin{tabular}{ll}
\hline Parameter & Value \\
\hline No. of neurons in the hidden layer & 20 \\
Learning rate & 0.72 \\
Momentum & 0.01 \\
No. of epochs & 10000 \\
\hline
\end{tabular}

in table 1. In RBF network, a classical two-stage learning method is applied. In the first stage, the values of centres were determined using a simple $k$-means clustering while the connection weights between the hidden neurons and the output layer were determined using least squared error. Empirically, we found out that the best performance of the RBF network is obtained with 12 clusters.

The actual against the predicted ozone concentrations based on the final developed MLP and $\mathrm{RBF}$ models for the area of Kopački Rit are shown 
in figures 6 and 7 , and the results for Osijek city are presented in figures 8 and 9 , respectively. The developed models are evaluated using the evaluation metrics listed in the previous section and compared in tables 2 and 3. From the evaluation results it can be noticed that MLP and RBF models for training and testing data are very competitive in the case of Kopački Rit area, whereas in the case of Osijek

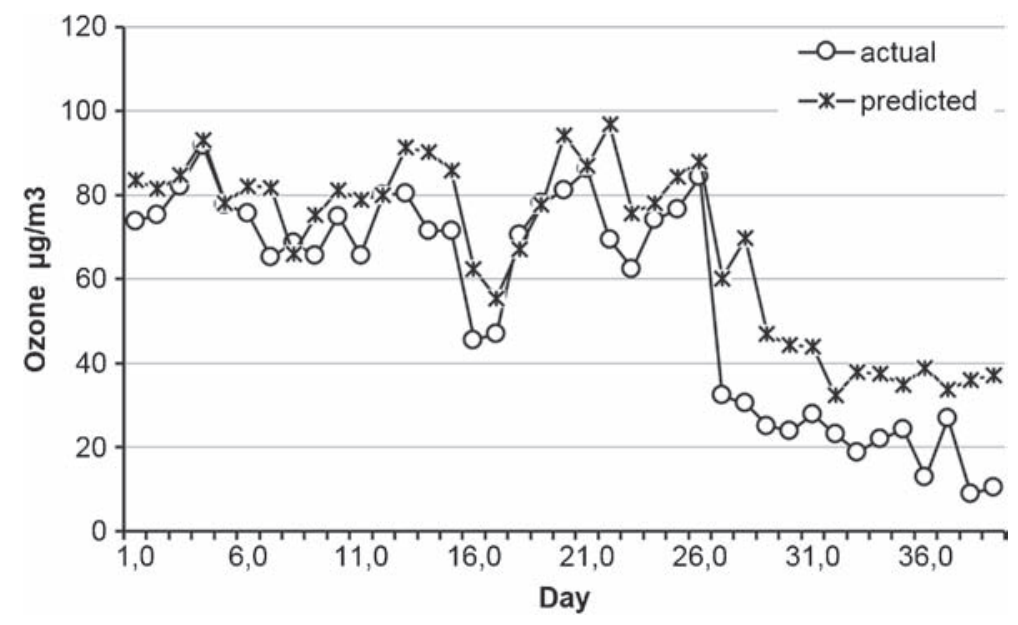

Figure 6. Actual vs. estimated values by MLP model for Kopački testing data.

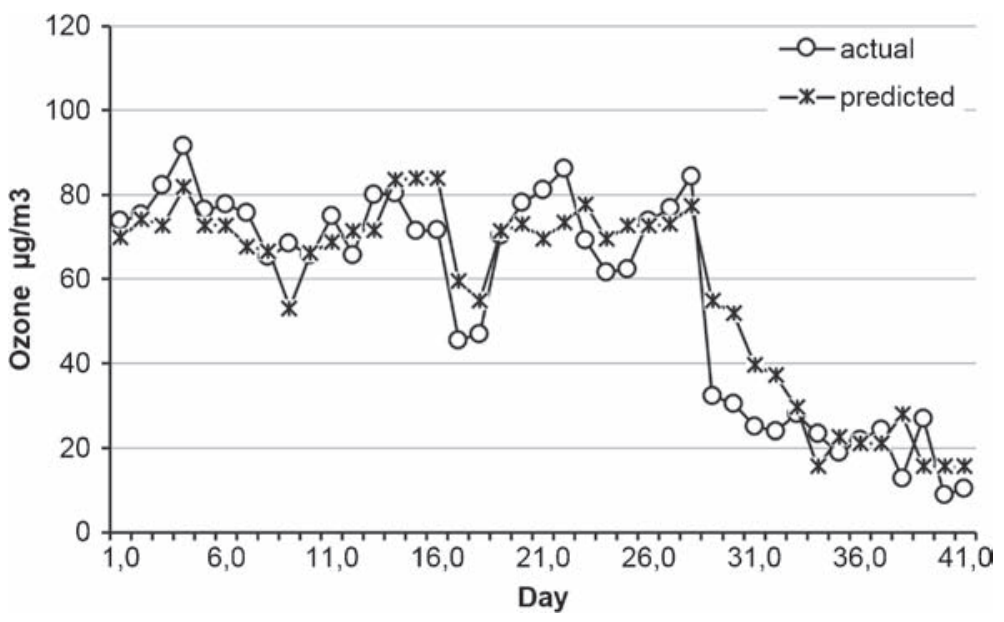

Figure 7. Actual vs. estimated values by RBF model for Kopački testing data.

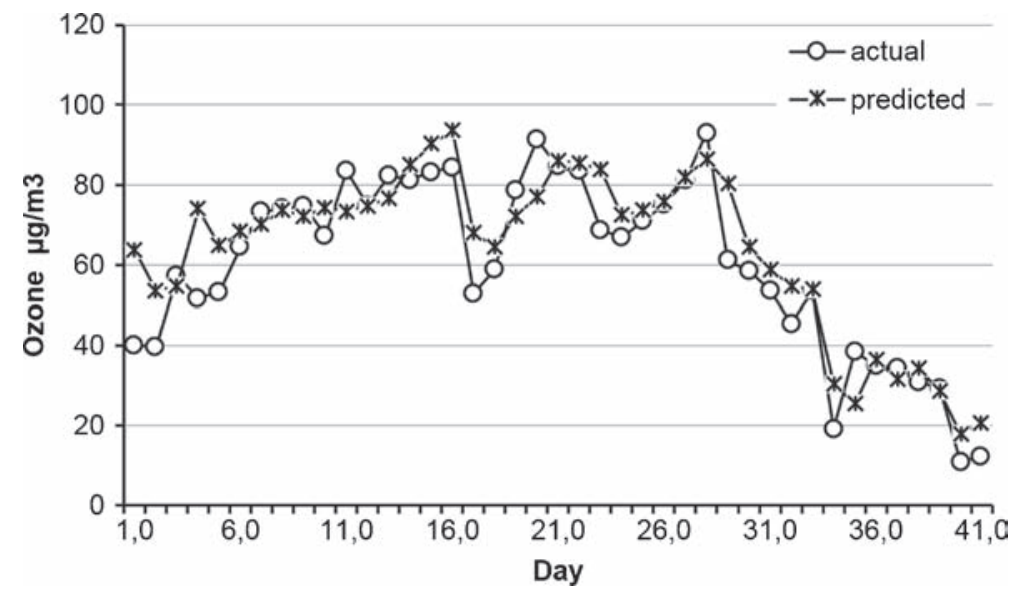

Figure 8. Actual vs. estimated values by MLP model for Osijek testing data. 


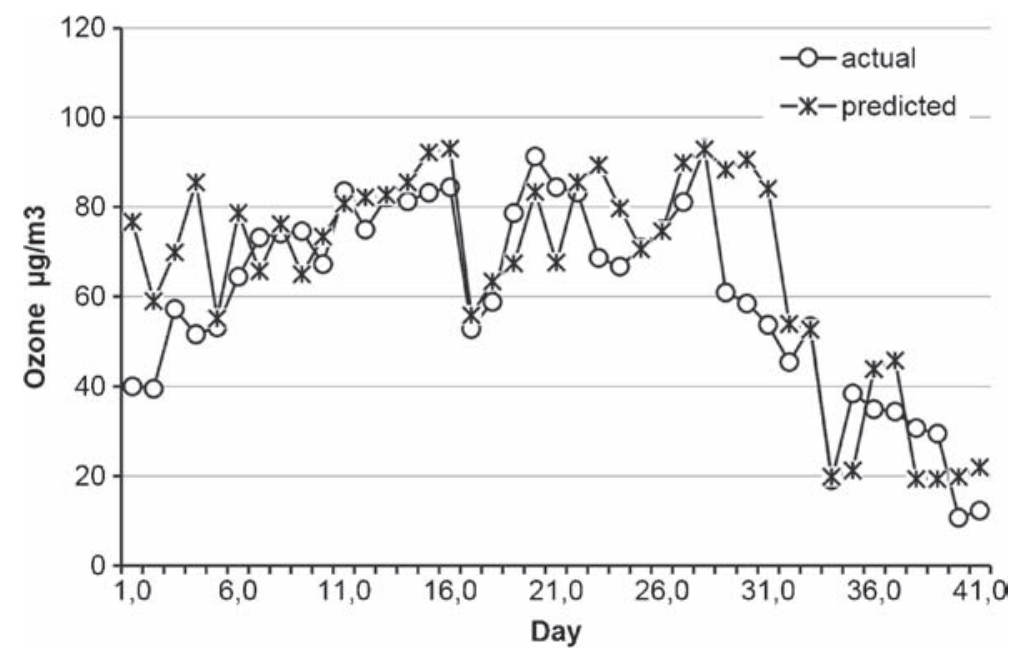

Figure 9. Actual vs. estimated values by RBF model for Osijek testing data.

Table 2. Evaluation results of $M L P$ and $R B F$ based on Kopački Rit data.

\begin{tabular}{lccccc}
\hline & \multicolumn{2}{c}{ MLP } & & \multicolumn{2}{c}{ RBF } \\
\cline { 2 - 3 } \cline { 6 - 6 } & Training & Testing & & Training & Testing \\
\hline$R^{2}$ & $90.18 \%$ & $92.02 \%$ & & $89.61 \%$ & $92.88 \%$ \\
VAF & $81.22 \%$ & $84.66 \%$ & & $80.29 \%$ & $86.08 \%$ \\
MSE & 302.03 & 86.103 & & 143.51 & 90.589 \\
MAE & 14.275 & 7.1294 & & 8.7649 & 7.8618 \\
RMSE & 17.379 & 9.2792 & & 11.98 & 9.5178 \\
\hline
\end{tabular}

Table 3. Evaluation results of $M L P$ and $R B F$ based on Osijek city data.

\begin{tabular}{lccccc}
\hline & \multicolumn{2}{c}{ MLP } & & \multicolumn{2}{c}{ RBF } \\
\cline { 2 - 3 } \cline { 6 - 6 } & Training & Testing & & Training & Testing \\
\hline$R^{2}$ & $92.58 \%$ & $92.02 \%$ & & $87.11 \%$ & $83.05 \%$ \\
VAF & $85.59 \%$ & 84.663 & & $75.89 \%$ & $63.56 \%$ \\
MSE & 122.25 & 86.103 & & 198.26 & 207.75 \\
MAE & 8.5345 & 7.1294 & & 11.216 & 10.801 \\
RMSE & 11.056 & 9.2792 & 14.08 & 14.414 \\
\hline
\end{tabular}

city, MLP shows better evaluation results with $9 \%$ improvement in the correlation coefficient.

Furthermore, a feature selection process is performed to find the best feature subset with the highest prediction power. The wrapper based feature selection method described earlier is applied on the training dataset of Osijek city and Kopački Rit. Since the total number of available features is small, we used an exhaustive search which searches the space of all possible subsets starting from the empty set of features.

All feature subsets selected by the exhaustive search are evaluated using an inductive algorithm
Table 4. Evaluation results of $M L P$ and $R B F$ based on Kopački Rit data after performing feature selection.

\begin{tabular}{lccccc}
\hline & \multicolumn{2}{c}{ MLP } & & \multicolumn{2}{c}{ RBF } \\
\cline { 2 - 3 } \cline { 5 - 6 } & Training & Testing & & Training & Testing \\
\hline$R^{2}$ & 0.9148 & 0.9274 & & 0.9585 & $92.57 \%$ \\
VAF & 0.8312 & 0.8569 & & 0.9185 & $84.73 \%$ \\
MSE & 128.96 & 95.67 & & 59.14 & 97.43 \\
MAE & 8.5237 & 8.0648 & & 5.6071 & 7.6379 \\
RMSE & 11.3559 & 9.781 & & 7.6903 & 9.8707 \\
\hline
\end{tabular}

Table 5. Evaluation results of $M L P$ and $R B F$ based on Osijek city data after performing feature selection.

\begin{tabular}{lccccc}
\hline & \multicolumn{2}{c}{ MLP } & & \multicolumn{2}{c}{ RBF } \\
\cline { 2 - 3 } \cline { 5 - 6 } & Training & Testing & & Training & Testing \\
\hline$R^{2}$ & 0.9203 & 0.902 & & 0.9497 & $88.68 \%$ \\
VAF & 0.8476 & 0.8125 & & 0.9016 & $77.66 \%$ \\
MSE & 131.39 & 98.51 & & 80.60 & 105.36 \\
MAE & 9.0928 & 7.6123 & & 6.8411 & 7.9859 \\
RMSE & 11.4626 & 9.9251 & & 8.9775 & 10.27 \\
\hline
\end{tabular}

and some evaluation function. In our experiments, MLP is selected as inductive algorithm while RMSE is chosen as an evaluation function. In order to increase the reliability of the results, a crossvalidation with three folds is applied in the process of training and testing the MLP network.

For the Kopački Rit case, the best feature subset obtained is $\left\{\mathrm{T}, \mathrm{RH}, \mathrm{WD}, \mathrm{O}_{3}(\mathrm{t}-1)\right\}$, while for the Osijek city is $\left\{\mathrm{RH}, \mathrm{WS}, \mathrm{O}_{3}(\mathrm{t}-1)\right\}$. The evaluation results of training and testing based on the selected features are summarised in tables 4 and 5 . According to these results, it can be noticed that the MLP network achieved very competitive results 
with those obtained previously based on all features. On the other hand, we can also notice that the feature selection process has improved the prediction power of RBF network.

\section{Conclusion}

In this work two artificial neural network models were developed for predicting the concentration of surface ozone in the east of Croatia. Ozone and $\mathrm{PM}_{10}$ levels were measured in two areas, the urban area of Osijek city and the rural area of Nature Reserve Kopački Rit. The obtained data indicate that those two pollutants as possible menace for the vegetation in Osijek, whereas in Kopački Rit they have no negative impact. More long-lasting studies are certainly needed to determine anthropogenic influences and transport of pollution as well as ozone injury on vegetation and human health.

The collected data for ozone levels were used for the development of MLP and RBF networks. Data was prepared by calculating the average of the ozone values for each day of the study period, whereas the first 25 days of each month were collected together and formed the training set while the last 5 days of each month formed the testing set. Empirical training of both networks gave the parameters for the best performance for MLP while the best performance in the case of RBF was obtained with 12 clusters. The evaluation results, in the case of Kopački Rit, showed that MLP and $\mathrm{RBF}$ models for training and testing data are very competitive. In the case of Osijek city, evaluation results for MLP revealed $9 \%$ improvement in the correlation coefficient.

Furthermore, the wrapper based feature selection method was used to find the best feature subset with the highest prediction power. The best feature subset obtained for Kopački Rit was $\{\mathrm{T}, \mathrm{RH}$, $\left.\mathrm{WD}, \mathrm{O}_{3}(\mathrm{t}-1)\right\}$ and for the Osijek city $\{\mathrm{RH}$, WS, $\left.\mathrm{O}_{3}(\mathrm{t}-1)\right\}$. Given the acquired results it can be concluded that the feature selection process has improved the prediction power of RBF network, while in the case of MLP network, achieved results were very competitive with those obtained previously based on all features.

\section{Acknowledgements}

The authors gratefully acknowledge the financial support given to the project by the Croatian Ministry of Science, Education and Sports. The authors also thank Meteorological and Hydrological Service of Croatia and the Ministry of Environmental and Nature Protection.

\section{References}

Agirre E et al. 2012 Forecasting ozone levels using artificial neural networks; In: Forecasting Models - Methods E Applications (ed.) Zhu J, Hong-Kong, iConcept Press Ltd., Chapter 14, pp. 207-219.

Alebić-Juretić A et al. 2007 Atmospheric particulate matter and ozone under heat-wave conditions: Do they cause an increase of mortality in Croatia? Bull. Environ. Contam. Toxicol. 79 468-471.

Alexis N et al. 2004 Health effects of air pollution; J. Allergy Clin. Immunol. 114 1116-1123.

Bytnerowicz A et al. 2007 Integrated effects of air pollution and climate change on forests: A northern hemisphere perspective; Environ. Pollut. 147 438-445.

Cape J N 2008 Surface ozone concentrations and ecosystem health: Past trends and a guide to future projections; Sci. Tot. Environ. 400 257-269.

Chen R et al. 2010 Ambient air pollution and hospital admission in Shanghai, China; J. Hazard. Mater. 181 $234-240$.

Directive 2002/3/EC of the European parliament and of the council of 12 February 2002 relating to ozone in ambient air, 2002.

Directive 2008/50/EC of the European parliament and of the council on ambient air quality and cleaner air for Europe, 2008.

Faris $\mathrm{H}$ et al. 2014 Artificial neural networks for surface ozone prediction: Models and analysis; Pol. J. Environ. Stud. 23 341-348.

Kalabokas P D et al. 2007 Vertical ozone measurements in the troposphere over the eastern Mediterranean and comparison with central Europe; Atmos. Chem. Phys. Discuss. 7 2249-2274.

Katsouyanni K et al. 2001 Confounding and effect modification in the short-term effects of ambient particles on total mortality: Results from 29 European cities within the APHEA2 project; Epidemiology 12 521531.

Kohavi R and John G H 1997 Wrappers for feature subset selection; Artificial Intelligence 97 273-324.

Kovač-Andrić E et al. 2013 Assessment of variations of $\mathrm{O}_{3}$ concentrations in Kopački Rit Nature Park, Eastern Croatia; Croat. Chem. Acta 86 109-115.

Ministry of Environmental Protection, 'Physical planning and environmental action plan' 2002.

Paoletti E 2009 Ozone and urban forests in Italy; Environ. Pollut. 157 1506-1512.

Pastor-Bárcenas O et al. 2005 Unbiased sensitivity analysis and pruning techniques in neural networks for surface ozone modelling; Ecol. Modell. 182 149-158.

Percy K E and Ferretti M 2004 Air pollution and forest health: Toward new monitoring concepts; Environ. Pollut. $130113-126$.

Pope C A III et al. 2002 Lung cancer, cardiopulmonary mortality, and long-term exposure to fine particulate air pollution; J. Amer. Med. Assoc. 297 1132-1141.

Prajapati S K and Triphati B D 2008 Seasonal variation of leaf dust accumulation and pigment content in plant species to urban particulates pollution; J. Environ. Quality 37 865-870.

Prybutok V R et al. 2000 Comparison of neural network models with ARIMA and regression models for prediction of houston's daily maximum ozone concentrations; European J. Oper. Res. 122 31-40. 
Saeys Y et al. 2007 A review of feature selection techniques in bioinformatics; Bioinformatics 23 2507-2517.

Sahu L K 2012 Volatile organic compounds and their measurements in the troposphere; Curr. Sci. 102 16451649.

Sahu L K and Saxena P 2015 High time and mass resolved PTR-TOF-MS measurements of VOCs at an urban site of India during winter: Role of anthropogenic, biomass burning, biogenic and photochemical sources; Atmos. Res. 164$16584-94$.

Salam T M et al. 2005 Birth outcomes and prenatal exposure to ozone, carbon monoxide, and particulate matter: Results from the Children's Health Study; Environ. Health Perspect. 113 1638-1644.
Sánchez-Lorenzo A et al. 2008 Spatial and temporal trends in sunshine duration over western Europe (1938-2004); J. Climate 21 6089-6098.

Stedman J R 2004 The predicted number of air pollution related deaths in the UK during the August 2003 heat wave; Atmos. Environ. 38 1087-1090.

UNESCO, Convention concerning the protection of the world structural and natural heritage, vol. 38, 2001.

Wittig V E et al. 2009 Quantifying the impact of current and future tropospheric ozone on tree biomass, growth, physiology and biochemistry: A quantitative meta-analysis; Global Change Biol. 15 396-424.

Yang W and Omaye S T 2009 Air pollutants, oxidative stress and human health; Mutation Research 674 45-54.

MS received 2 December 2015; revised 26 February 2016; accepted 23 March 2016

Corresponding editor: K KRISHNAMOORTHY 